\title{
La dynamique des avalanches d'après les résultats des expériences de déclenchement artificiel au Lautaret*
}

PAR

\author{
J. L. Allera, G. Bon-Mardion', A. Eybert-Bérard', \\ C. Guelff, R. Mura", P. Perroud', L. Rey" \\ (1) Centre d'Etudes Vucléaires de Grenoble, Laboratoire d'Applications Spéciales de la Physique \\ (2) Dixision de Nivologie du CTGREF (Ministère de l'Agriculture) \\ (3) Centre d'Etudes de la Neige (Météorologie Nationale) \\ 33 - Saint-Murtin d'Hères
}

\section{Introduction}

A la demande de la Division Nivologie du C.T.G.R.E.F. de St-Martin d'Hères (Isère) et avec sa collaboration ainsi que celle du Centre d'Etudes de la Neige (Météorologie Nationale de St Martin d'Hères), nous poursuivons, dans le cadre du programme "Dynaneige", les mesures dynamiques dans l'avalanche entreprises depuis 1973 sur le site expérimental du Col du Lautaret (Hautes-Alpes). Nous présentons les résultats acquis au cours de la saison de neige 1975. Ce document complète la note CENG/ASP $n^{\circ} 75-01$ : "Mesures dynamiques dans l'avalanche. Résultats expérimentaux de la saison de neige 1973-1974" $[1,2]$. Tous les déclenchements sont faits à l'explosif, le câble transporteur d'explosif s'étant avéré un moyen très commode et efficace. La mise en ouvre est réalisée par un exploseur de sécurité étudié spécialement et mis au point par le Laboratoire A.S.P. [3].

\section{Installation expérimentale}

Par rapport à l'installation déjà existante, diverses modifications ont été apportées en 1975 (fig. 1).

a) Sur chacun des deux nouveaux couloirs, des consolessupports de capteurs ont été implantés. Ce sont les couloirs 6 et 7 sur la face Nord-Est de la crête de Chaillol. Ces deux

(*) Ce travail se place dans le cadre du contrat GR-759.949 avec la Division de Nivologie du Centre Technique du Génie Rural et des Eaux et Forêts de St Martin d'Hères (38400) couloirs sont desservis comme les autres sur la face SudEst par le câble transporteur d'explosif (fig. 2).

b) Le couloir $n^{\circ} 1$ a été équipé d'un système de mesure de la masse volumique de l'aérosol de l'avalanche.

c) Les supports des moulinets "Neyrflux" ont été modifiés afin qu'ils puissent recevoir une sonde de platine pour les mesures de la neige pendant l'écoulement.

d) Les consoles $A$ et $B$ peuvent recevoir une rallonge supportant un anémomètre destiné à la mesure de vitesse de l'aérosol de l'avalanche.

e) Enfin, un deuxième abri de mesures a été construit près du premier, il est destiné a l'entrepôt des matériels d'expérience.

\section{Méthode de mesures}

La mesure de la vitesse des particules dans l'avalanche. de la force d'impact et de la masse volumique a été déjà décrite [1]. Il s'agit de moulinets hydrauliques, de capteurs à jauges de contraintes et de la technique d'atténuation $\gamma$. Des mesures nouvelles ont été inaugurées.

\subsection{Masse volumique $\rho(t)$ de l'aérosol de l'avalanche}

Le principe utilisé est le même que pour la mesure de la masse volumique de l'avalanche, c'est-à-dire l'atténuation d'un rayonnement $\gamma$ par la matière. Les masses volumiques considérées étant dans ce cas bien plus faibles (1 $\mathrm{kg} / \mathrm{m}^{3}$ ), il s'est avéré nécessaire de faire des essais 


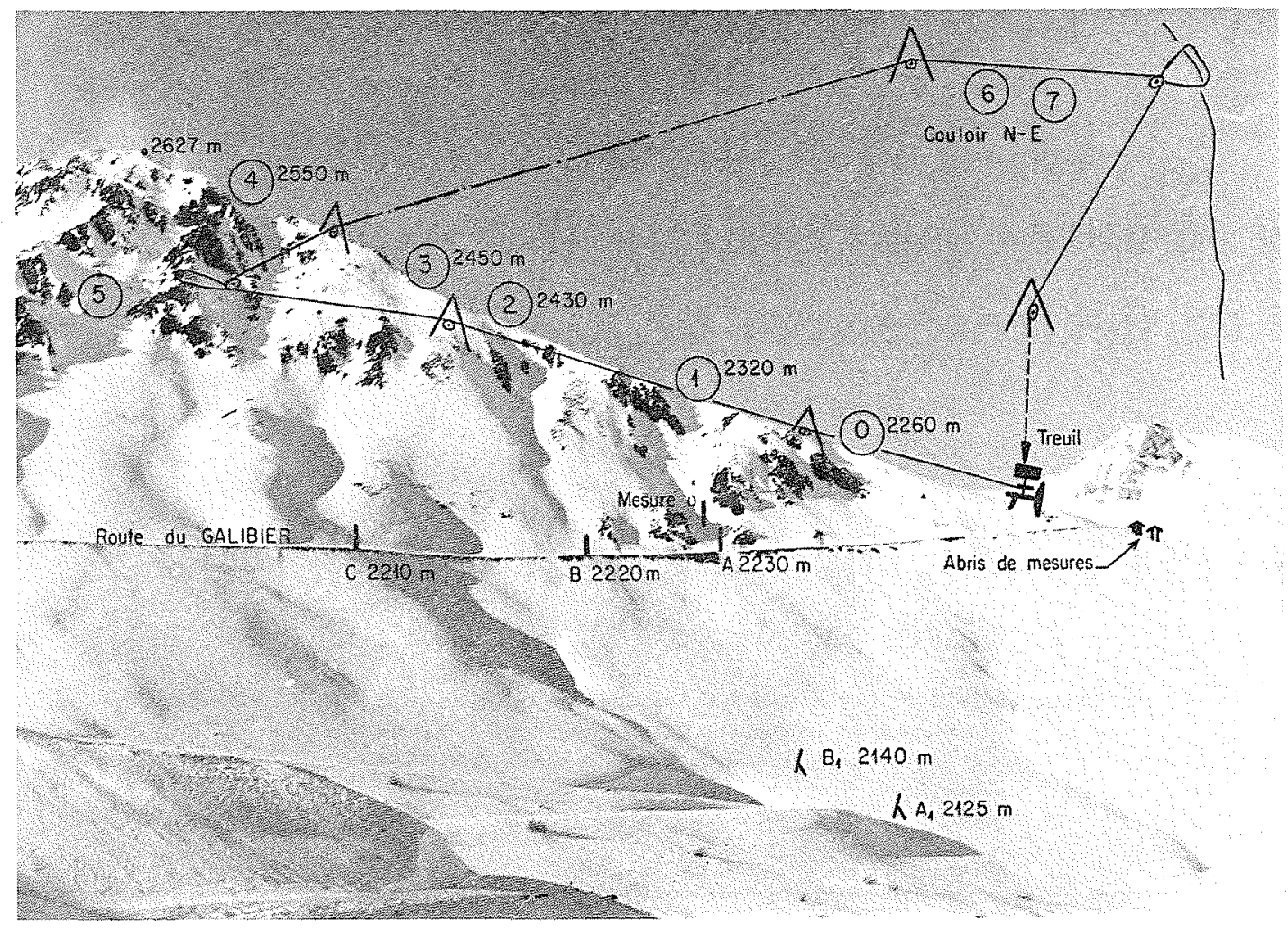

1/ Site expérimental du Col du Lautaret $(05)$ - Crête de Chaillol

préalables en laboratoire. Ceux-ci nous ont conduits au choix des éléments suivants :

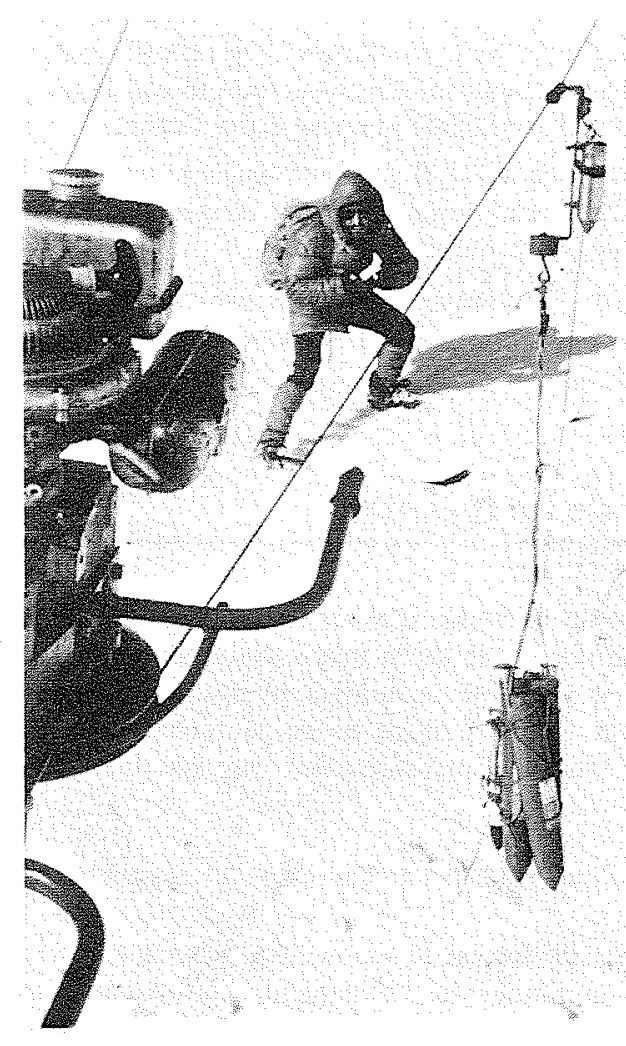

2/ Câble transporteur d'explosif muni de l'exploseur de sécurité.
- source $\gamma$ : caesium 137

- activité : $37 \mathrm{mCi}$

- seuil : $300 \mathrm{keV}$

- temps de réponse : $0,4 \mathrm{~s}$

- distance source : PM 2,24 m

L'appareillage est monté sur un équipage mobile supporté par un câble tendu au-dessus du couloir $n^{\circ} 1$ à une hauteur de $5 \mathrm{~m}$ environ au-dessus du fond (fig. 3 ).

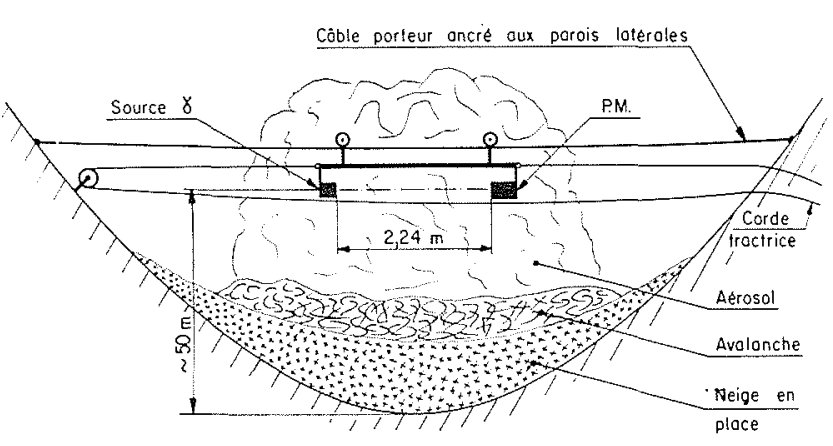

$3 /$ Profil transversal du couloir $n^{\bar{j}} 1$ au niveau de la mesure $\gamma$ aérosol.

Synoptique de la mesure :

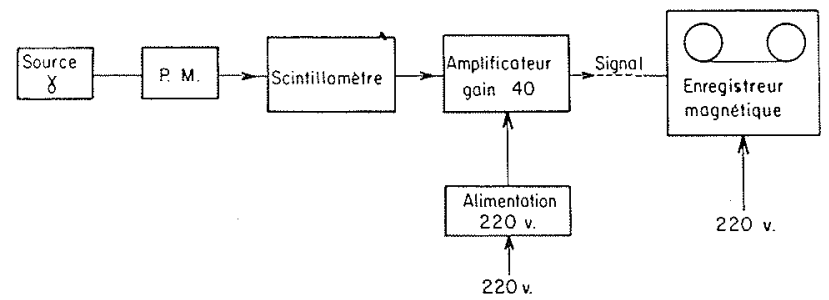




\subsection{Température de la neige en écoulement}

Sur les consoles $A$ et $B$ des couloirs $n^{\circ} 1$ et 2 , nous avons installé un dispositif de mesure de température en continu de la neige. L'élément sensible est une sonde de fil de platine, protégée mécaniquement par un doigt en acier inoxydable assurant un bon contact thermique. La sonde est insérée sur une branche d'un pont de Wheatstone dont le signal de sortie est proportionnel aux variations de résistance du fil de platine, donc aux variations de température. Un système de compensation permet d'éliminer l'erreur due aux grandes longueurs de câble de mesure utilisées (environ $300 \mathrm{~m}$ ) (fig. 4).

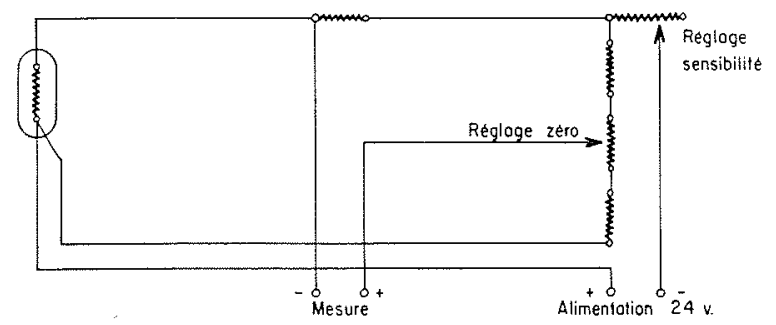

4/Principe de la mesure.

L'alimentation du pont est assurée par des batteries $24 \mathrm{~V}$ au cadmium-nickel de bonne stabilité :

- sensibilité de la sonde $: 1 \mathrm{mV} /{ }^{\circ} \mathrm{C}$

- courant traversant la sonde : $2,8 \mathrm{~mA}$

- erreur due aux variations de la résistance de ligne : $<0.1 \%$ pour une ligne d'une résistance de $20 \Omega$.

Les inconvénients de cette méthode de mesure sont d'une part le temps de réponse de la sonde $(0,4 \mathrm{~s})$ et d'autre part la nécessité d'étalonner in situ donc dans des conditions défavorables.

Synoptique de la mesure :

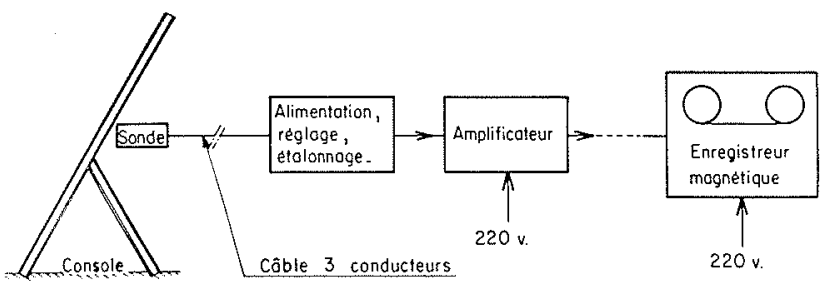

\subsection{Vitesse de l'aérosol de l'avalanche}

Points de mesure : console A ou B.

Méthode anénomètre directionnel à hélice, type W 106 de la Météorologie Nationale.

Principe : un capteur délivre, par l'intermédiaire d'un disque métallique crénelé défilant entre les bobines d'entretien d'un circuit oscillant, des impulsions électriques dont la fréquence varie avec la vitesse de rotation de l'hélice. Ces impulsions sont ensuite mises en forme et intégrées. Il est à noter que la discrimination de la direction a été supprimée, l'anémomètre étant fixé dans l'axe de l'écoulement. D'autre part, l'écoulement de l'aérosol étant turbulent, nous ne prendrons en compte que les variations des fronts de montée des impulsions.

Synoptique de la mesure :

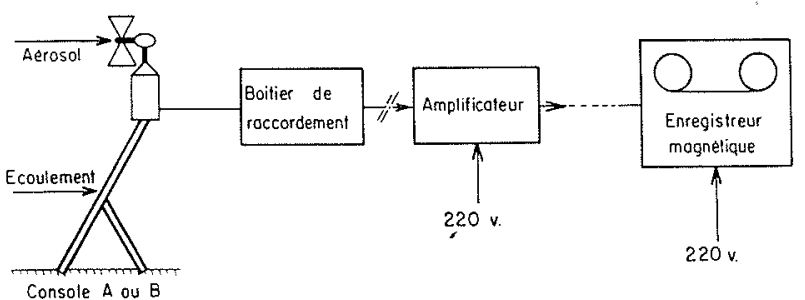

\subsection{Vitesse du front, épaisseur et importance de l'ava- lanche}

Des balises constituées par des piquets métalliques surmontés de panneaux de toile rouge et jaune ont été implantées entre les couloirs $n^{\circ} 1$ et 2 dans l'axe de l'écoulement.

Un appareil photographique "Nikon $F_{2}$ " à moteur électrique, assurant une cadence de prises de vues de 4 images/s, placé perpendiculairement à l'axe de l'écoulement, permet de suivre l'avalanche sur une grande partie de son trajet avec un objectif approprié.

Le relevé topographique des balises, de l'appareil, des couloirs $n^{\circ} 1$ et 2 réalisé par l'équipe du C.T.G.R.E.F. d'une part et le dépouillement graphique du film d'autre part permettent de connaitre avec une précision suffisante la vitesse du front et l'importance de l'avalanche (fig. 5).

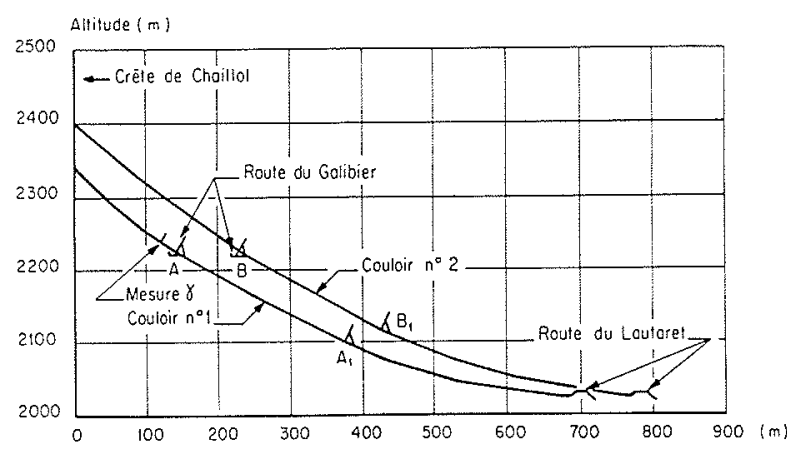

$5 /$ Profil en long des couloirs $n^{\circ} 1$ et $n^{\circ} 2$ (d'après CTGREF, Avril 1974)

\subsection{Charges électrostatiques transportées par l'avalanche}

Pour tenter d'expliquer le phénomène qui lors d'une avalanche de poudreuse produit un claquement sec, nous avons essayé de mettre en évidence l'apparition des charges électrostatiques dans la neige sèche et froide.

Un essai a eu lieu lors du déclenchement du 22 Janvier 1975, la mesure a été effectuée à l'aide d'un voltmètre électronique "Rotschild" placé à quelques mètres du bord de l'écoulement. Une faible électrisation a été notée au passage de l'avalanche, mais sans accumulation des charges. Les conditions d'électrisation n'étaient sans doute pas réunies. 


\section{Résultats expérimentaux}

Cinq avalanches importantes ont été ainsi provoquées venant s'ajouter aux sept autres des années précédentes, ce qui porte le nombre total à douze. Le tableau 1 donne une récapitulation sommaire.

Des mesures complètes concernant chacune des avalanches sont données en annexe. Il est à remarquer que l'avalanche cataloguee "poudreuse" est en fait une avalanche de neige sèche, froide, assez dense, surmontée d'un nuage d'aérosol.

\section{Remarques:}

a) La mesure de la masse volumique de l'aérosol par atténuation de rayonnement $\gamma$ n'a pas encore donné de résultats positifs pour les raisons suivantes:
- pas ou très peu d'aérosol lors des avalanches des 21 Janvier et 12 Mars,

- très faible atténuation lors de l'avalanche du 31 Janvier. La résolution du système de mesure n'a pas permis son exploitation.

b) Les mesures de températures par sonde de platine ont été très souvent perturbées par l'écoulement (rupture de la sonde), un nouveau système de mesure est en cours de réalisation.

c) L'enneigement médiocre n'a pas permis de déclencher beaucoup d'avalanches et le nombre des mesures a été insuffisant.

d) Les hauteurs de neige mentionnées au sommet des couloirs ne correspondent pas toujours aux hauteurs relevées sur les sondages, ceci vient du fait que les sondages sont réalisées, pour des raisons de sécurité, hors des couloirs.

\begin{tabular}{|c|c|c|c|c|c|c|c|c|c|c|c|c|c|c|}
\hline \multicolumn{15}{|c|}{$\begin{array}{l}\text { Site expérimental du col du Lautaret. } \\
\text { Tableau récapitulatif des avalanches : couloirs } 1 \text { et } 2(1973,1974,1975) \text {. }\end{array}$} \\
\hline \multirow[t]{2}{*}{ Couloirs } & \multirow[t]{2}{*}{ Date } & \multirow[t]{2}{*}{$\begin{array}{l}\text { Aspect de } \\
\text { l'avalanche }\end{array}$} & \multirow{2}{*}{$\begin{array}{c}\text { Epaisseur } \\
\text { moyenne } \\
\text { de la neige } \\
\text { en place } \\
{[\mathrm{m}]}\end{array}$} & \multirow{2}{*}{$\begin{array}{c}\text { Epais. de } \\
\text { la cassure } \\
\text { de départ } \\
{[\mathrm{m}]}\end{array}$} & \multicolumn{2}{|c|}{$\begin{array}{c}\text { Températures } \\
{\left[{ }^{\circ} \mathrm{C}\right]}\end{array}$} & \multicolumn{2}{|c|}{$\begin{array}{l}\text { Vitesses } \\
\text { (au po } \\
{[\mathrm{m} / \mathrm{s}]}\end{array}$} & \multicolumn{3}{|c|}{$\begin{array}{l}\text { Masse volumique } \\
\text { nt de mesure) } \\
{\left[\mathrm{kg} / \mathrm{m}^{3}\right]}\end{array}$} & \multicolumn{3}{|c|}{$\begin{array}{c}\text { Pressions } \\
\text { dynamiques } \\
\text { [bar] }\end{array}$} \\
\hline & & & & & Air & $\begin{array}{l}\text { Sur- } \\
\text { face } \\
\text { neige }\end{array}$ & $\max$ & moy. & $\begin{array}{c}\rho_{o} \\
\text { avant }\end{array}$ & $\begin{array}{c}\rho \\
\text { après }\end{array}$ & $\rho / \rho_{o}$ & $\max$ & moy. & $\frac{P_{\max }}{P_{\operatorname{moy}}}$ \\
\hline $\begin{array}{l}\qquad N^{\circ} 1 \\
\text { Alt. départ } 2320 \mathrm{~m} \\
\text { Alt. mesure } 2230 \mathrm{~m} \\
\text { Dénivellation } 90 \mathrm{~m} \\
\text { Nature : herbe rase, } \\
\text { roche, éboulis } \\
\text { Orientation S.E. }\end{array}$ & $\begin{array}{l}24.01 .73 \\
14.02 .73 \\
08.02 .74 \\
01.03 .74 \\
22.01 .75 \\
31.01 .75 \\
12.03 .75\end{array}$ & $\begin{array}{l}\text { Plaque } \\
\text { Poudreuse } \\
\text { Poudreuse } \\
\text { Poudreuse } \\
\text { Poudreuse } \\
\text { Poudreuse } \\
\text { Poudreuse } \\
\text { et plaque } \\
\text { à vent }\end{array}$ & $\begin{array}{l}1,00 \\
1,85 \\
1,00 \\
1,10 \\
2,03 \\
2,50 \\
2,50\end{array}$ & $\begin{array}{l}0,40 \\
0,30 \\
0,65 \\
0,30 \\
0,35 \\
0,50 \\
0,30\end{array}$ & $\begin{array}{r}-5,8 \\
-10,0 \\
-\quad 9,8 \\
0 \\
+\quad 0,8 \\
-\quad 3,0\end{array}$ & $\begin{array}{c}-9,6 \\
-\quad 6,4 \\
-10,1 \\
-\quad 8,1 \\
0 \\
-\quad 3,2 \\
-\quad 4,0\end{array}$ & $\begin{array}{l}15,5 \\
26,0 \\
16,5 \\
13,5 \\
16,8\end{array}$ & $\begin{array}{r}8,0 \\
12,0 \\
\simeq 7,4 \\
\simeq 7,0\end{array}$ & $\begin{array}{r}260 \\
110 \\
81 \\
151 \\
225 \\
184 \\
312\end{array}$ & $\begin{array}{l}300 \\
165 \\
195 \\
246 \\
276 \\
271 \\
396\end{array}$ & $\begin{array}{l}1,15 \\
1,43 \\
2,41 \\
1,63 \\
1,2 \\
1,5 \\
1,3\end{array}$ & $\begin{array}{l}0,46 \\
0,65 \\
1,30 \\
0,40 \\
0,46 \\
1,40 \\
1,30\end{array}$ & $\begin{array}{l}0,16 \\
0,24 \\
0,50 \\
0,20 \\
0,20 \\
0,40 \\
0,40\end{array}$ & $\begin{array}{l}2,5 \\
2,7 \\
2,6 \\
2,0 \\
2,0 \\
3,5 \\
3,2\end{array}$ \\
\hline $\begin{array}{l}\qquad \mathrm{N}^{\circ} 2 \\
\text { Alt. départ } 2430 \mathrm{~m} \\
\text { Alt. mesure } 2220 \mathrm{~m} \\
\text { Dénivellation } 210 \mathrm{~m} \\
\text { Nature : herbe rase, } \\
\text { roche, éboulis } \\
\text { Orientation S.E. }\end{array}$ & $\begin{array}{l}24.01 .73 \\
14.02 .73 \\
08.02 .74 \\
22.01 .75 \\
12.03 .75\end{array}$ & $\begin{array}{l}\text { Plaque } \\
\text { Poudreuse } \\
\text { Poudreuse } \\
\text { Poudreuse } \\
\text { Poudreuse } \\
\text { et plaque } \\
\text { à vent }\end{array}$ & $\begin{array}{l}0,70 \\
1,85 \\
1,00 \\
2,30 \\
2,80\end{array}$ & $\begin{array}{l}0,30 \\
0,25 \\
0,65 \\
0,30 \\
0,30\end{array}$ & $\begin{array}{l}-3,0 \\
-\quad 2,2\end{array}$ & $\begin{array}{l}-8,2 \\
-9,0 \\
-8,4 \\
-4,6 \\
-1,5\end{array}$ & $\begin{array}{l}16,5 \\
28 \\
16 \\
19\end{array}$ & $\begin{array}{r}9,0 \\
11,0 \\
\approx 6,0 \\
11\end{array}$ & $\begin{array}{r}260 \\
115 \\
80 \\
225 \\
272\end{array}$ & $\begin{array}{l}300 \\
259 \\
246 \\
301 \\
332\end{array}$ & $\begin{array}{l}1,15 \\
2,25 \\
3,08 \\
1,3 \\
1,2\end{array}$ & $\begin{array}{l}0,08 \\
1,16 \\
2,90 \\
1,02 \\
1,20\end{array}$ & $\begin{array}{l}0,04 \\
0,40 \\
1,10 \\
0,50 \\
0,30\end{array}$ & $\begin{array}{l}2,0 \\
2,9 \\
2,6 \\
2,0 \\
4,0\end{array}$ \\
\hline
\end{tabular}

\section{Références bibliographiques}

[1] Bon Mardion (G.), Coche (G.), Eybertberard (A.), JoURdAN (P.), PERROUd (P.), REY (L.) - Mesures dynamiques dans l'avalanche. Premiers résultats expérimentaux. Note CENG/ASP n ${ }^{\circ} 74-01$, Mars 1974.

[2] Bon Mardion (G.), Eybert-berard (A.), Guelff (C.), PERROUD (P.), REX (L.) - Mesures dynamiques dans l'avalanche. Résultats expérimentaux de la saison de neige 19731974

Note CENG/ASP n ${ }^{\circ} 75-01$, Février 1975.

[3] Laboratoire A.S.P./C.E.N.G. - Division Nivologie/C.T.G.R.E.F. Câble transporteur d'explosif pour le déclenchement des avalanches.

Note CENG/ASP n ${ }^{\circ} 74-10$, Novembre 1974

\section{ANNEXE 1}

\section{AVALANCHES DU COULOIR $\mathrm{N}^{\circ} 1$}

\section{Avalanche du 22 Janvier 1975}

1. Conditions nivo-météorologiques à $14 \mathrm{~h}$

Etat du couloir :

Epaisseur totale de neige dans l'axe du couloir et au sommet : $203 \mathrm{~cm}$, dont 20 à $25 \mathrm{~cm}$ de neige récente.

Au niveau de la console $A$, épaisseur totale de neige $230 \mathrm{~cm}$, dont 40 à $50 \mathrm{~cm}$ de neige récente et transportée par le vent (fig. 6). 


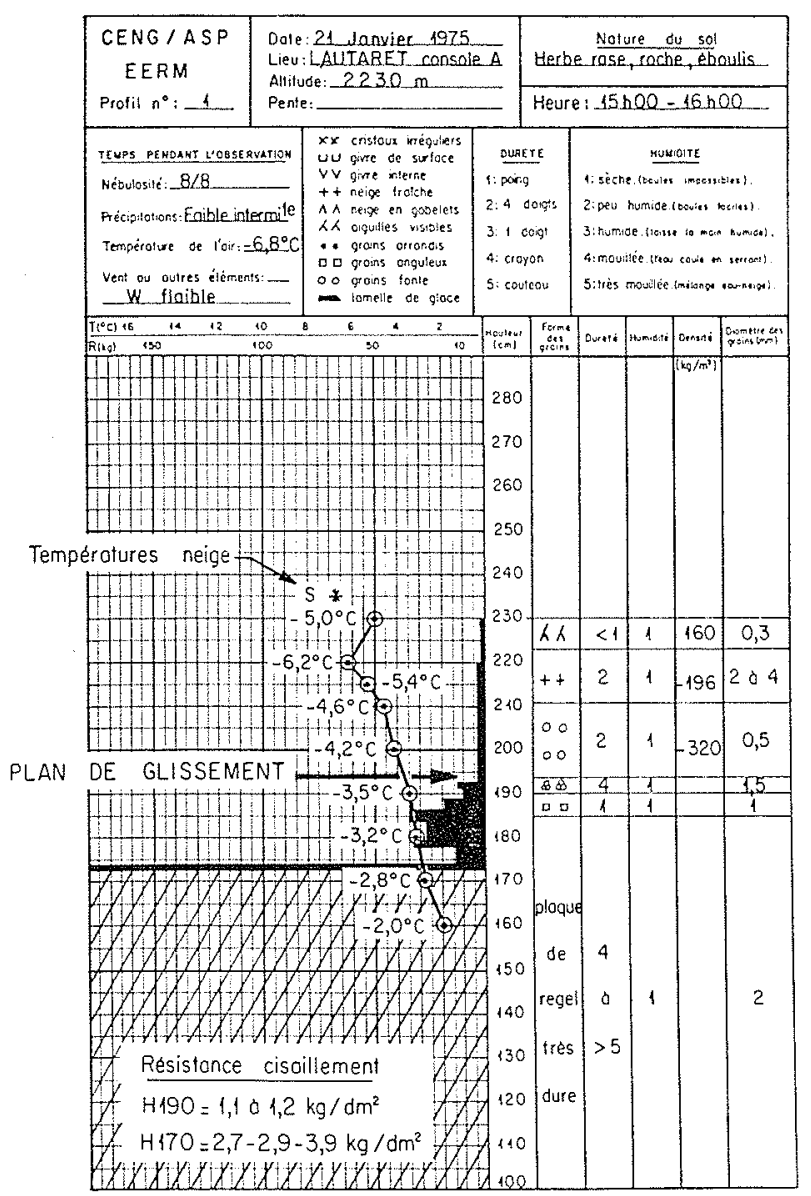

6/Etat initial de la neige.

Qualité de la neige de surface:

Sèche, un peu soufflée, grains ou parties de cristaux de $\emptyset=0.3 \mathrm{~mm}$. Plusieurs niveaux de neige roulée, avec cristaux intacts visibles dans ces strates. Au-dessous, croûte de regel assez dure, sur plaque de vieille neige très dure.

\section{Résistance au cisaillement .}

A la base de la couche de surface, la résistance au cisaillement est de l'ordre de $1 \mathrm{~kg} / \mathrm{dm}^{2}$. Au-dessous, la résistance au cisaillement, dans la plaque de neige dure, dépasse $3 \mathrm{~kg} / \mathrm{dm}^{2}$.

\section{Plan de glissement :}

Le plan de glissement possible doit se situer à la base de la couche de surface.

\section{Température neige de surface:}

La température superficielle de la neige dans le couloir $n^{\circ} 1$ est de $0^{\circ} \mathrm{C}$ avant le déclenchement.

\section{Température air :}

La température de l'air est voisine de $0^{\circ} \mathrm{C}$.

\section{Caractéristiques de l'avalanche}

\section{a) Zone de départ:}

Epaisseur de la cassure : $25 \mathrm{~cm}$ environ.

\section{b) Zone intermédiaire : Console $A$}

Epaisseur de neige transportée après passage avalanche à l'axe du couloir : 25 à $35 \mathrm{~cm}$.

Granulométrie : Nombreuses boules 05 à $10 \mathrm{~cm}$. Débris de blocs provenant de la corniche du sommet (20 à $40 \mathrm{~cm}$ de côté).

Remarque : Les boules et blocs sont soudés entre eux en moins de $30 \mathrm{~mm}$

\section{c) Zone d'arrêt:}

L'avalanche s'arrête au niveau de la console Bl (alt. $2140 \mathrm{~m}$ ). La largeur du dépôt est d'environ 15 à $20 \mathrm{~m}$, l'épaisseur de neige de 30 à $40 \mathrm{~cm}$.

Présence de nombreuses boules de $\emptyset 10 \mathrm{~cm}$. Quelques blocs de $1 \mathrm{~m}^{3}$, provenant d'une plaque détachée sous la route du Galibier sont visibles à la zone d'arrêt.

\section{Résultats expérimentaux}

L'examen de l'enregistrement de la pression d'impact montre deux phases dans l'avalanche (fig. 7) : une phase correspondant à la tête de l'avalanche avec des pics de pression très rapprochés, une autre phase avec une pres-
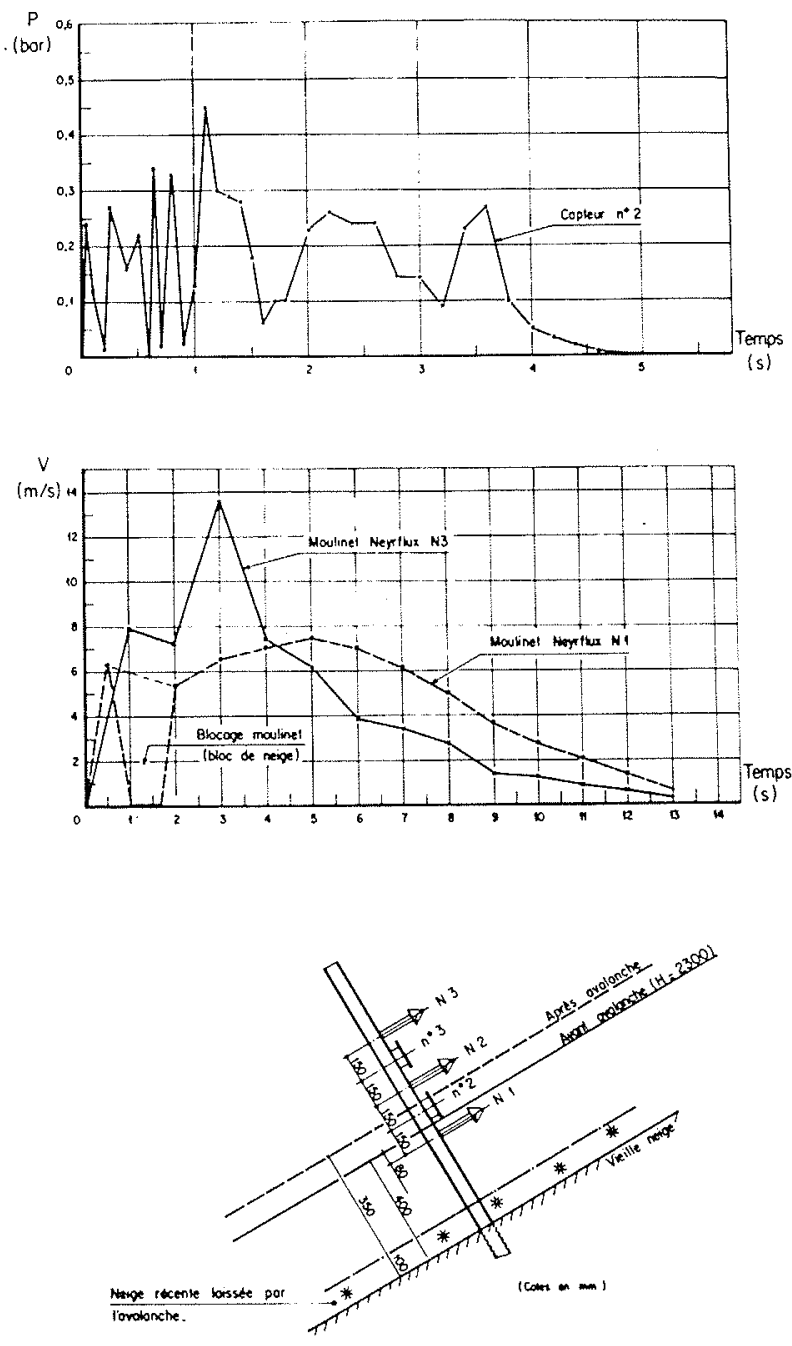

$7 /$ Site du Lautaret le 22 Janvier 1975 - Couloir $n^{\circ} 1-$ Console A 
sion moyenne plus importante, correspondant à un écoulement plus régulier $d u$ corps de l'avalanche. La figure 7 bis illustre bien ce phénomène.

La pression maximale enregistrée est de 0,45 bar, pendant un écoulement de 5 secondes.
La vitesse maximale mesurée est $13,5 \mathrm{~m} / \mathrm{s}$. L'appareil Nikon n'a pas fonctionné, le temps brumeux avec nappes de brouillard n'autorisait pas les prises de vues.

La mesure de la masse volumique par atténuation de rayonnement $\gamma$ (fig. 8) montre une décroissance régu-

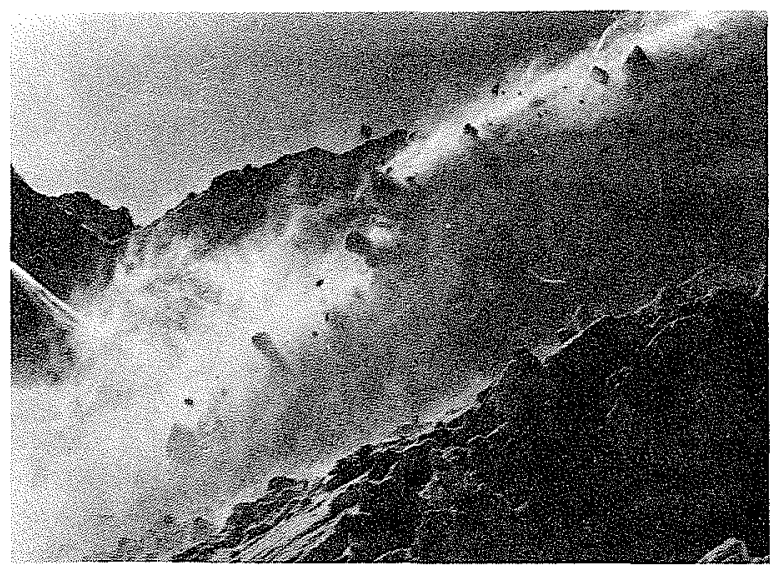

Photo a : Téte de l'avalanche. Notez la présence de blocs de neige.

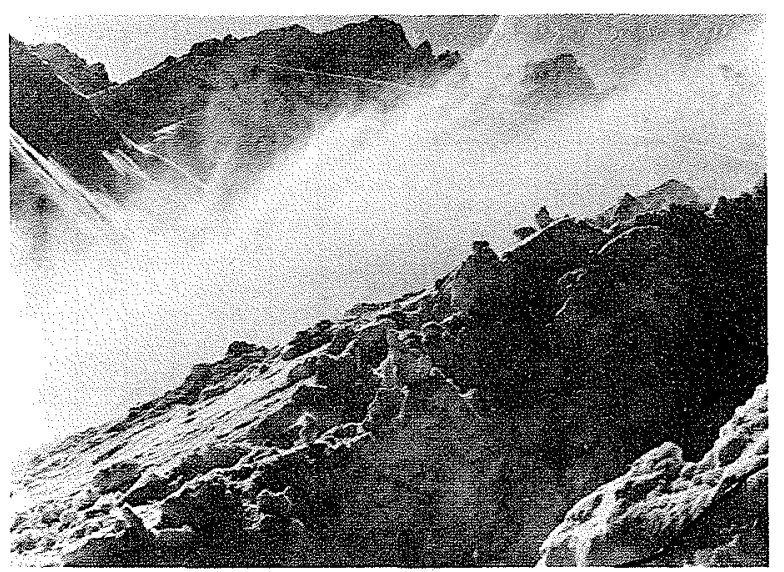

Photo $b$ : Corps de l'avalanche. La photo $b$ est prise 3 secondes après la photo $a$.

7 bis/ Site expérimental du Col du Lautaret. Passage de l'avalanche de poudreuse du 22 Janvier 1975 sur la console A (couloir $n^{\circ} 1$ ) - La hauteur de la neige en mouvement est environ $3 \mathrm{~m}-$

Chocs/Seconde (c/s)
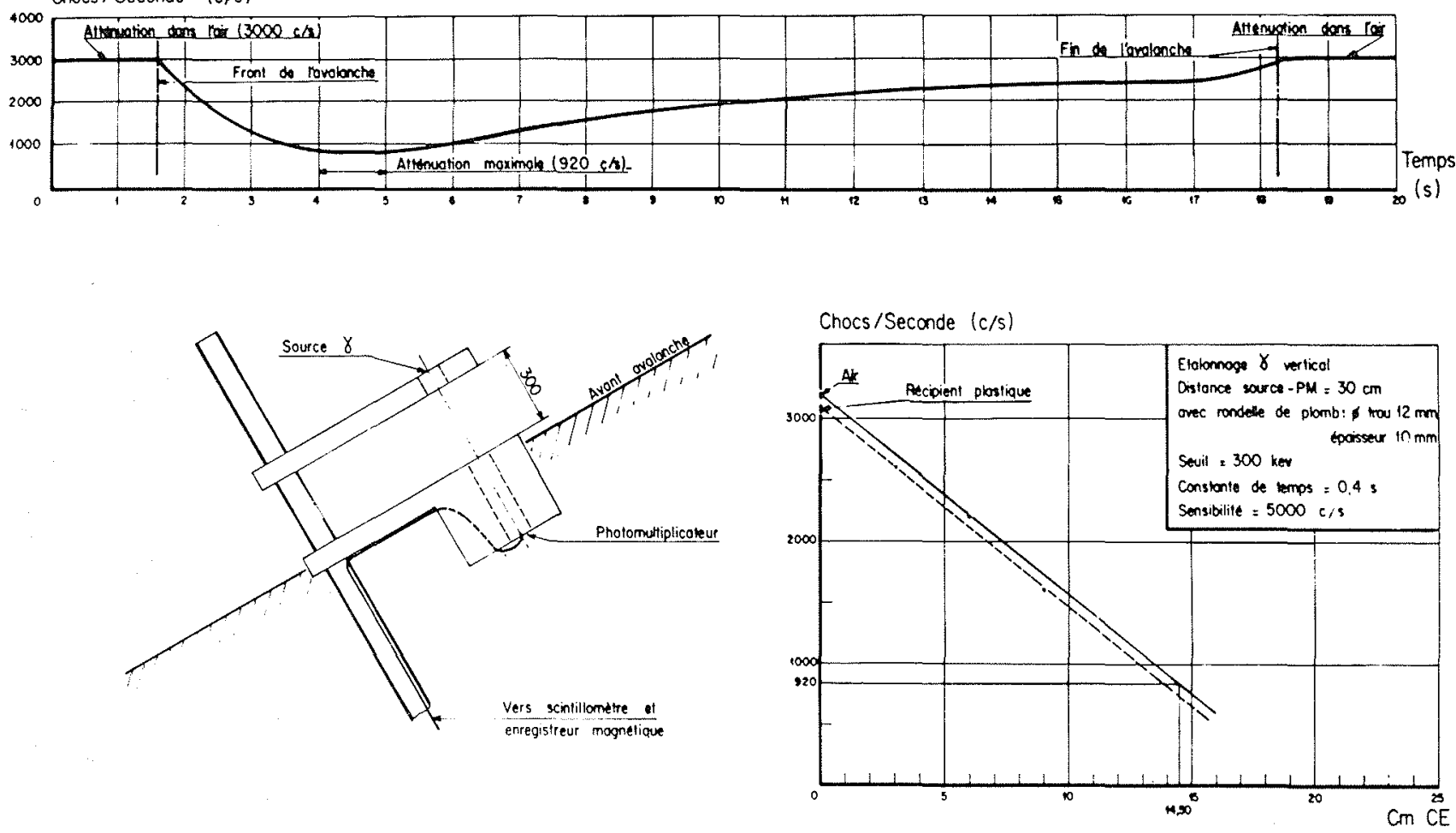

$8 /$ Site du Lautaret le 22 Janvier 1975 - Couloir $n^{\circ} 1$ Mesure de l'équivalent en eau de l'avalanche 
G. BON MARDION et al

lière jusqu'à un palier ( 920 chocs/s); l'atténuation étant maximale, l'avalanche recouvre complètement le capteur de mesures. L'épaisseur de neige est donc de $30 \mathrm{~cm}$ d'où d'après la courbe d'étalonnage, on a :

$$
\rho=\frac{14,5}{30}=0,480 \text { soit } 480 \mathrm{~kg} / \mathrm{m}^{3}
$$

Cette valeur semble assez élevée comparée aux mesures faites avant et après l'avalanche (voir tableau ci-dessous).

\begin{tabular}{|c|c|c|c|c|}
\cline { 3 - 5 } \multicolumn{1}{c|}{} & \multicolumn{2}{c|}{$\begin{array}{c}\text { Masse volumique } \\
{\left[\mathrm{kg} / \mathrm{m}^{3}\right]}\end{array}$} & \multicolumn{2}{c|}{ Températures $\left[{ }^{\circ} \mathrm{C}\right]$} \\
\hline Niveaux & Avant & Après & Avant & Après \\
\hline surface & 160 & 296 & $-0,0$ & $-0,0$ \\
$-5 \mathrm{~cm}$ & - & 316 & - & $-1,0$ \\
$-10 \mathrm{~cm}$ & 196 & 240 & $-3,2$ & $-2,5$ \\
$-20 \mathrm{~cm}$ & 320 & 252 & $-4,0$ & $-1,8$ \\
$-30 \mathrm{~cm}$ & & & $-3,0$ & $-2,2$ \\
\hline$\rho$ moyen & 225 & 276 & $-2,6$ & $-1,5$ \\
\hline
\end{tabular}

\section{Avalanche du 31 Janvier 1975}

\section{Conditions nivo-météorologiques à $10 \mathrm{~h}$}

\section{Etat du couloir:}

Epaisseur totale de neige au sommet : environ $250 \mathrm{~cm}$ dont 100 à $120 \mathrm{~cm}$ de neige fraîche due aux chutes des 28 et 29 Janvier.

Au niveau de la console $A$, épaisseur totale de neige environ $280 \mathrm{~cm}$ (chutes cumulées avec les culots d'avalanches antérieures) ; épaisseur de neige récente : environ $20 \mathrm{~cm}$ (fig. 9).

Qualité de la neige de surface au sommet:

Neige sèche, faible croûte en surface sur $2 \mathrm{~cm}$, audessous $8 \mathrm{~cm}$ de cristaux brisés par le vent $\emptyset \simeq 0,3 \mathrm{~mm}$. Au-dessous, plaque compacte composée de petits grains arrondis de $0,3 \mathrm{~mm}$, formée par le vent avec quelques niveaux peu épais de neige roulée, grains de $\emptyset 1,0 \mathrm{~mm}$.

\section{Qualité de la neige de surface niveau console $A$ :}

Neige sèche avec faible croûte de regel de 1 à $2 \mathrm{~cm}$ sur 30 à $35 \mathrm{~cm}$ de neige composée de particules reconnaissables, de dureté 1 , sur couche plus compacte de petits grains de $\emptyset 0,5 \mathrm{~mm}$.

Plan de glissement probable :

$\mathrm{Au}$ départ, il devrait se situer à la base de la plaque à vent qui doit entrainer la couche de $30 / 35 \mathrm{~cm}$ de neige de faible cohésion qui occupe le couloir dans la partie médiane (niveau console 1).

\section{Température neige :}

$$
-3,2^{\circ} \mathrm{C} \text { à } 11 \text { h } 20 \text { au niveau de la console } \mathrm{A} \text {. }
$$

\section{Température air :}

$$
-0,8^{\circ} \mathrm{C} \text {. }
$$

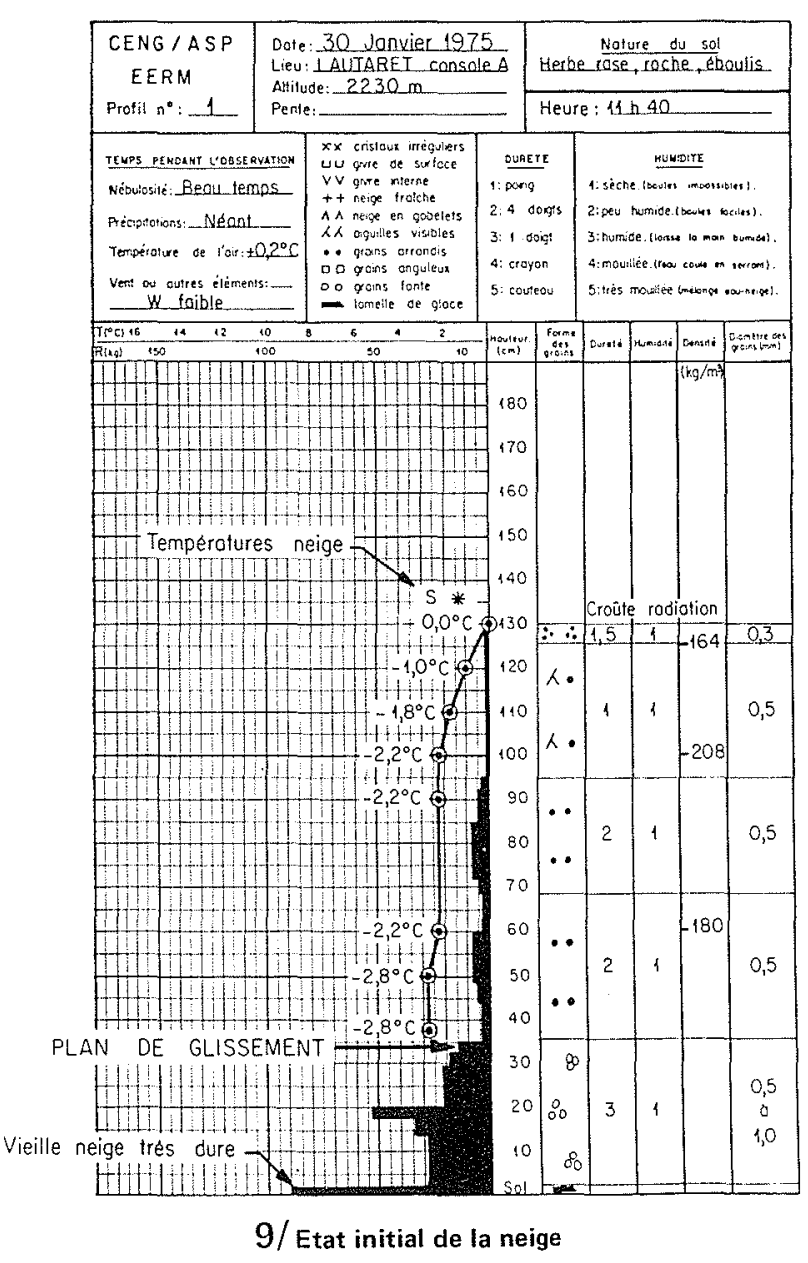

\section{Caractéristiques de l'avalanche}

\section{a) Zone de départ:}

Epaisseur de la cassure : 40 à $50 \mathrm{~cm}$ environ sur toute la largeur du couloir.

\section{b) Zone intermédiaire : Niveau console A}

Epaisseur de neige après avalanche 50 à $90 \mathrm{~cm}$ supplémentaires dans le couloir au niveau des capteurs.

Hauteur du nuage de neige poudreuse au niveau de la mesure $\gamma$ : environ $2,50 \mathrm{~m}$.

Granulométrie : majorité de petits grains arrondis $\phi<0,5 \mathrm{~mm}$ ou groupes de grains soudés de $0,2 / 0,3 \mathrm{~mm}$ de $\varphi$ (aspect neige roulée). Blocs peu nombreux, de dimensions comprises entre 5 et $20 \mathrm{~cm}$.

Remarque : La plaque de neige au départ n'était que de dureté 2 à 3 .

\section{c) Zone d'arrêt.}

Etalement à $15 \mathrm{~m}$ environ au-dessous de la console A1. Largeur du dépôt : 25 à $30 \mathrm{~m}$. Epaisseur 40 à $120 \mathrm{~cm}$ au centre du cône. Quelques blocs de dimensions $50 \times 50 \mathrm{~cm}$. Majorité de boules de $\phi \simeq 10 \mathrm{~cm}$.

\section{Résultats expérimentaux}

Pression d'impact : mème constatation que pour l'avalanche du 22 Janvier, à savoir un début d'écoulement per- 
turbé pendant les premiers dixièmes de seconde, ensuite un écoulement plus régulier. On distingue en plus deux fronts séparés à $t=2,4 \mathrm{~s}$ (Fig. 10 ).

Pression maximale : capteur haut : 1,4 bar, capteur bas : 1,0 bar.

Le coefficient d'augmentation de la masse volumique est de 1,5 .

L'élévation maximale de la température est de $+0,9^{\circ} \mathrm{C}$

\begin{tabular}{|c|c|c|c|c|}
\cline { 3 - 5 } \multicolumn{1}{c|}{} & \multicolumn{2}{c|}{$\begin{array}{c}\text { Masse volumique } \\
{\left[\mathrm{kg} / \mathrm{m}^{3}\right]}\end{array}$} & \multicolumn{2}{c|}{ Températures [ $\left.{ }^{\circ} \mathrm{C}\right]$} \\
\hline Niveaux & Avant & Après & Avant & Après \\
\hline surface & 164 & 240 & $-3,2$ & $-2,0$ \\
$-10 \mathrm{~cm}$ & - & - & $-2,8$ & $-1,8$ \\
$-20 \mathrm{~cm}$ & 208 & 260 & $-3,0$ & $-2,2$ \\
$-30 \mathrm{~cm}$ & - & - & $-2,2$ \\
$-40 \mathrm{~cm}$ & 180 & 312 & $-2,8$ & $-2,4$ \\
$-50 \mathrm{~cm}$ & & & $-2,2$ \\
\hline$\rho$ moyen & 184 & 271 & $-3,0$ & $-2,1$ \\
\hline
\end{tabular}

Coeff. augm. $=1,5 \quad \Delta t^{\circ} \mathrm{C}=+0,9^{\circ} \mathrm{C}$
Résistances au cisaillement :

- neige de surface (particules reconnaisables) $R=100$ à $120 \mathrm{~g} / \mathrm{dm}^{2}$

- $-10 \mathrm{~cm}$ (petits grains, bonne cohésion) $R=2000$ à $3000 \mathrm{~g} / \mathrm{dm}^{2}$

$-20 \mathrm{~cm}$ à $3500 \mathrm{~g} / \mathrm{dm}^{2}$

\section{Avalanche du 12 Mars 1975}

1. Conditions nivo-météorologiques

Etat du couloir

Epaisseur totale de neige : environ $250 \mathrm{~cm}$ dont 30 à $35 \mathrm{~cm}$ de neige récente.

$\mathrm{Au}$ niveau de la console $\mathrm{A}$, épaisseur totale de neige : environ $158 \mathrm{~cm}$ dont $30 \mathrm{~cm}$ de neige récente. Il est à noter qu'au niveau de la console A, l'épaisseur du manteau neigeux est extrêmement fluctuante du fait des avalanches précédentes et de l'effet du vent (fig. 11).
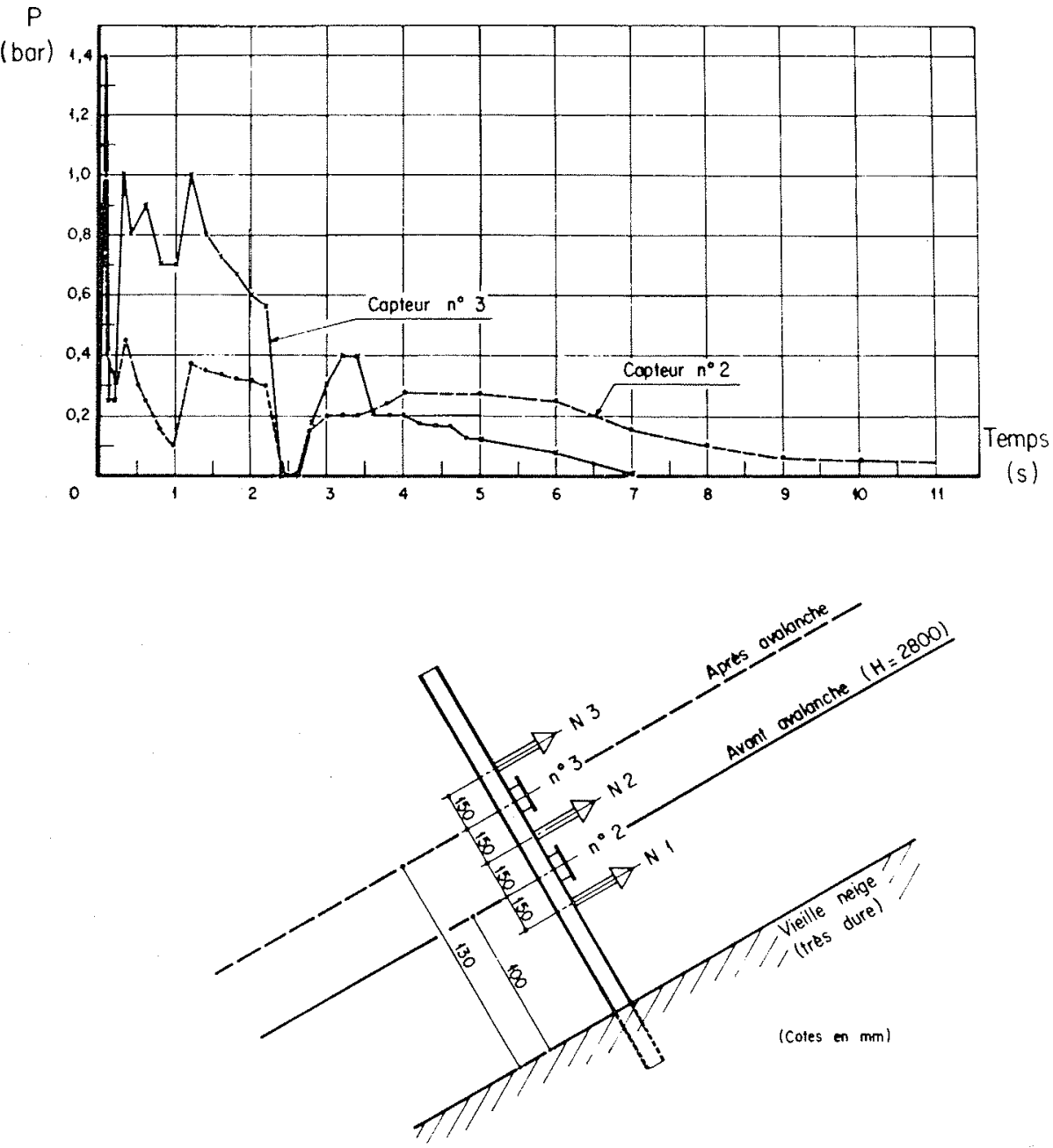

10/ Site du Lautaret le 31 Janvier $1975-$ Couloir $n^{\circ} 1-$ Console A 


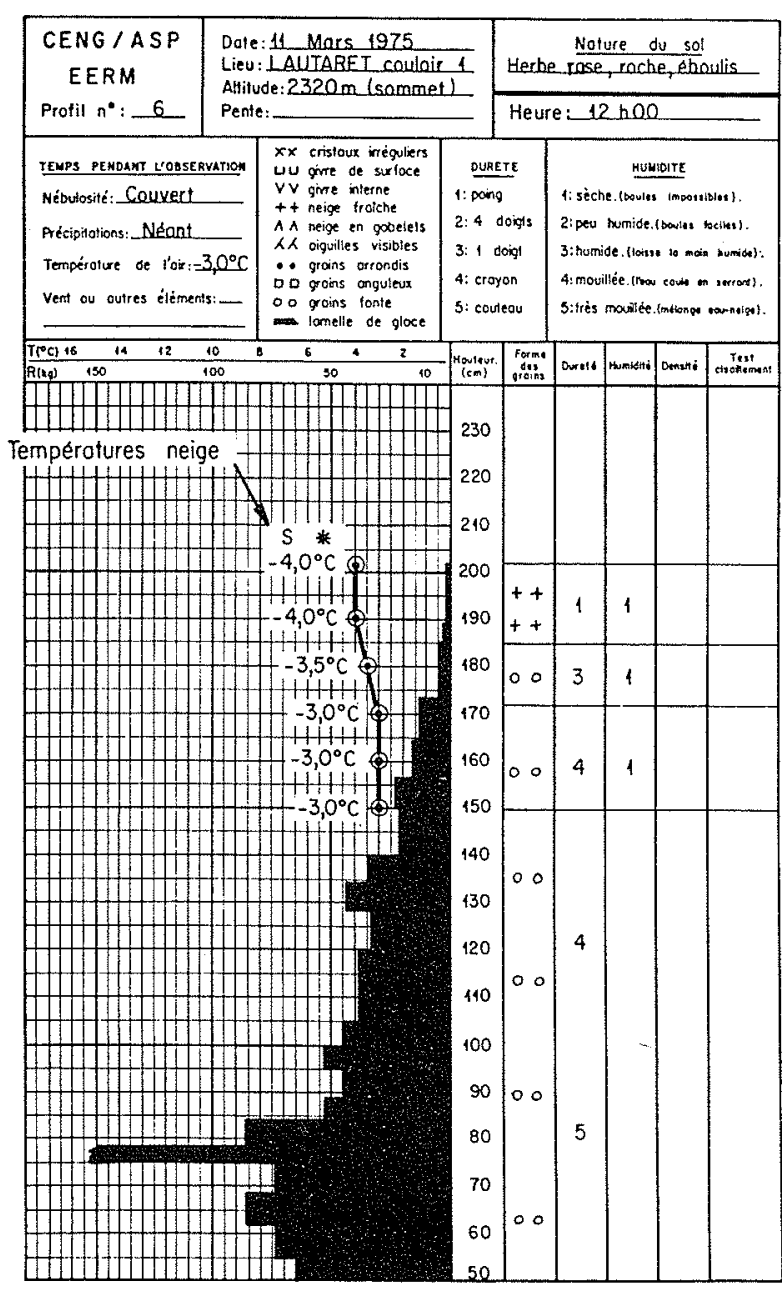

$11 /$ Etat initial de la neige
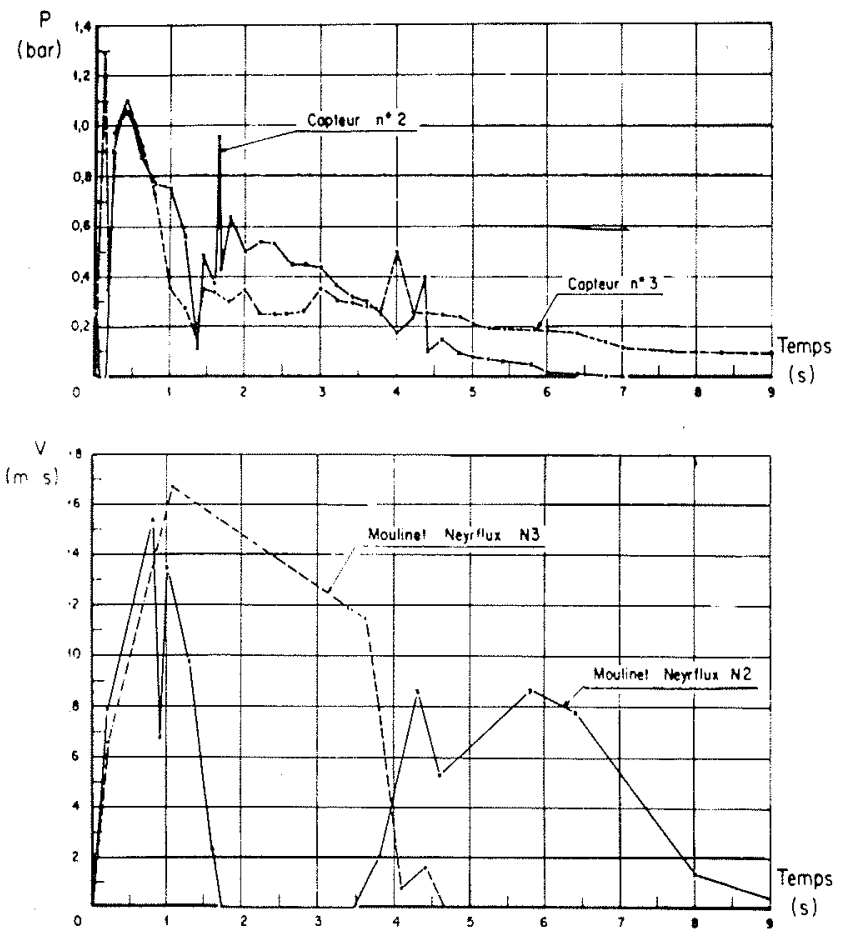

Qualité de la neige de surface au sommet :

Neige sèche, cohésion faible dans les 30 premiers centimètres, beaucoup plus dure au-dessous ; grains fins soudés.

Qualité de la neige de surface : Niveau console A

Neige sèche, cohésion et dureté faibles sur $30 \mathrm{~cm}$.

Au-dessous vieille neige compacte.

Plan de glissement probable:

L'avalanche doit intéresser uniquement la couche de surface, sur une épaisseur moyenne de $30 \mathrm{~cm}$.

Température neige en surface.

$$
-4,0^{\circ} \mathrm{C}(09 \mathrm{~h})
$$

Température air : (non notée)

2. Caractéristiques de l'avalanche

a) Zone de départ:

Epaisseur probable de la cassure 20 à $30 \mathrm{~cm}$

b) Zone intermédiaire : Console A

Apport supplémentaire de $30 \mathrm{~cm}$ de neige aux capteurs. Epaisseur de neige remuée par l'avalanche au même point : environ $45 \mathrm{~cm}$.

c) Zone d'arrêt : Manque de renseignements.

\section{Résultats expérimentaux}

On note toujours le méme phénomène en début d'écoulement. Les pressions d'impact ont leur maximum à l'arrivée du front, avec un pic correspondant à un deuxième front (fig. 12).

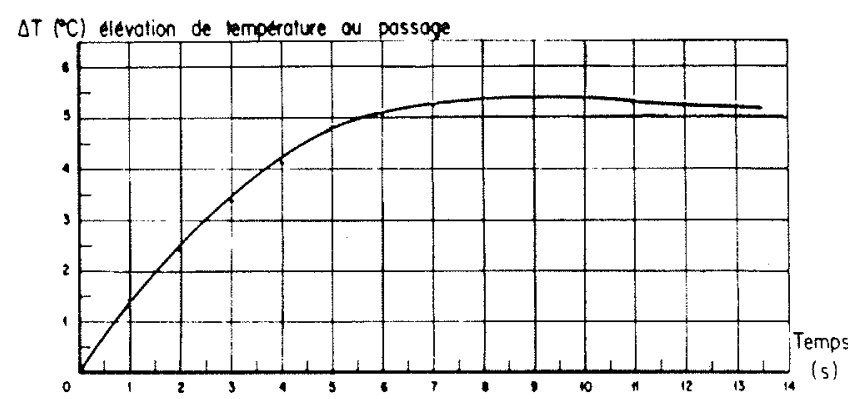

12/ Site du Lautaret le 12 Mars 1975 - Couloir $n^{\circ} 1$ Console A 
Pression maximale : capteur haut 1,05 bar capteur bas 1,30 bar.

Les vitesses d'écoulement, bien que perturbées, donnent tout de même une idée des vitesses.

Vitesse maximale : capteur haut $16,8 \mathrm{~m} / \mathrm{s}$ capteur bas $15,2 \mathrm{~m} / \mathrm{s}$

Masse volumique moyenne avant avalanche : $312 \mathrm{~kg} / \mathrm{m}^{3}$ " " " après " : $396 \mathrm{~kg} / \mathrm{m}^{3}$

Augmentation des températures : $+1,6^{\circ} \mathrm{C}$.

\begin{tabular}{|c|c|c|c|c|}
\cline { 2 - 5 } \multicolumn{1}{c|}{} & \multicolumn{2}{c|}{$\begin{array}{c}\text { Masse volumique } \\
{\left[\mathrm{kg} / \mathrm{m}^{3}\right]}\end{array}$} & \multicolumn{2}{c|}{ Températures $\left[{ }^{\circ} \mathrm{C}\right]$} \\
\hline Niveaux & Avant & Après & Avant & Après \\
\hline surface & 140 & 360 & $-4,0$ & $-1,5$ \\
$-10 \mathrm{~cm}$ & 240 & 340 & $-3,0$ & $-1,0$ \\
$-20 \mathrm{~cm}$ & 400 & 420 & $-2,5$ & $-0,5$ \\
$-30 \mathrm{~cm}$ & 380 & 440 & $-1,5$ & 0 \\
$-40 \mathrm{~cm}$ & 400 & 420 & 0 & 0 \\
\hline$\rho$ moyen & 312 & 396 & $-2,2$ & $-0,6$ \\
\hline
\end{tabular}

Coeff. augm. $=1.3 \Delta t^{\circ} \mathrm{C}=+1,6^{\circ} \mathrm{C}$

La mesure de l'élévation de température pendant l'écoulement (Fig. 12) montre un $\Delta t$ de $6^{\circ} \mathrm{C}$. L'imprécision de l'étalonnage in situ apporte quelques réserves sur la position du zéro. D'autres mesures demandent confirmation du phénomène.

\section{ANNEXE 2}

\section{AVALANCHES DU COULOIR $\mathrm{N}^{\circ} 2$}

\section{Avalanche du 22 Janvier 1975}

1. Conditions nivo-météorologiques (Fig. 13)

\section{Etat du couloir :}

Epaisseur totale de neige : environ $200 \mathrm{~cm}$ dont $30 \mathrm{~cm}$ de neige fraiche en moyenne. Les valeurs d'enneigement au sommet et la qualité de la neige sont similaires à celles relevées au couloir $\mathrm{n}^{\circ} 1$ le même jour.

$\mathrm{Au}$ niveau de la console $\mathrm{B}$, épaisseur totale de neige : $164 \mathrm{~cm}$ dont 25 à $30 \mathrm{~cm}$ de neige fraiche.

Qualité de la neige de surface :

Neige légèrement ventée, peu de particules reconnaissables, grains de $\phi \leqslant 0,5 \mathrm{~mm}$.

Au-dessous, neige transformée en une couche compacte de densité élevée : $320 \mathrm{~kg} / \mathrm{m}^{3}$.

Plan de glissement probable:

A la base de la couche de neige fraiche. Peu de possibilité pour que l'avalanche détruise la croûte dure de surface de la vieille neige.

Températures neige en surface:

$$
-4,6^{\circ} \mathrm{C} \text {. }
$$

Température air :

$$
-2,2{ }^{\circ} \mathrm{C} \text {. }
$$

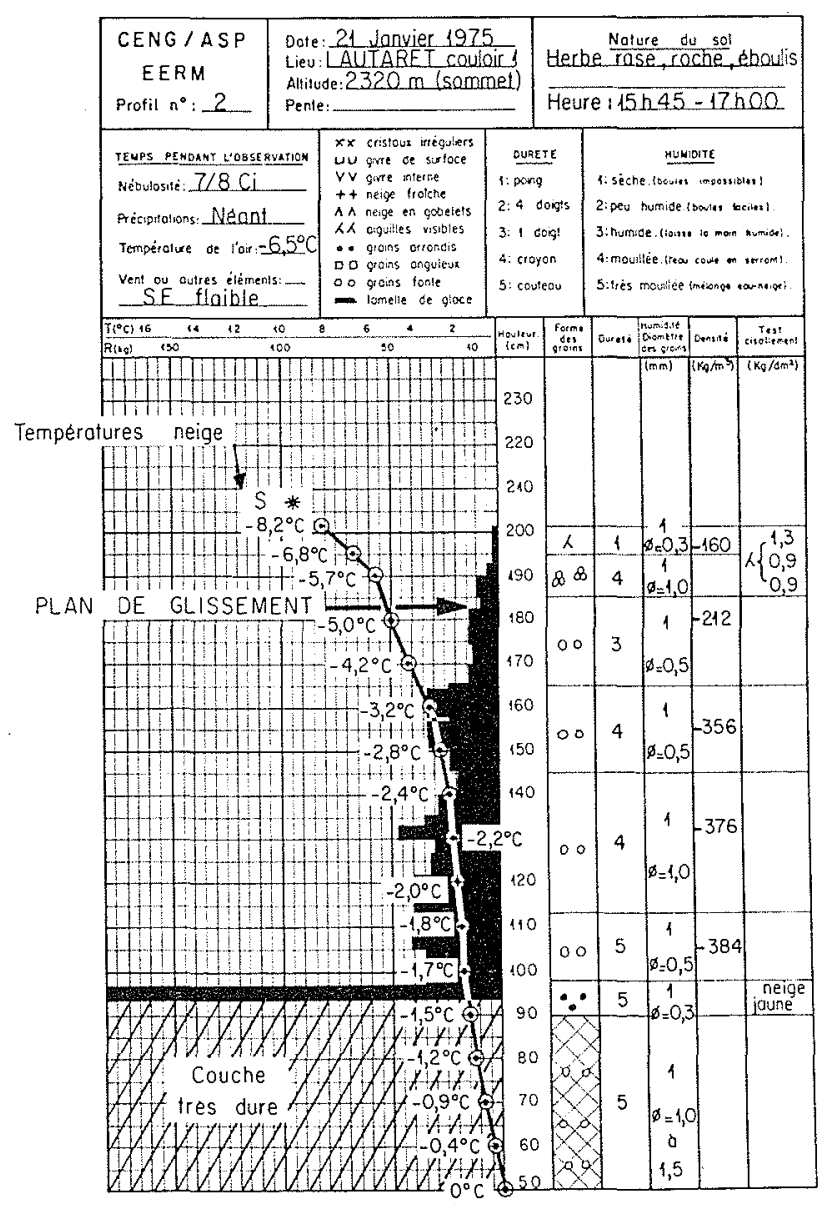

13/ Etat initial de la neige

\section{Caractéristiques de l'avalanche}

a) Zone départ :

Epaisseur de la cassure : environ $25 \mathrm{~cm}$.

b) zone intermédiaire :

Epaisseur neige transportée au niveau de la console B : environ $50 \mathrm{~cm}$.

Grains de $\emptyset \leqslant 0,5 \mathrm{~mm}$. Nombreuses boules de $\phi 10 \mathrm{~cm}$.

c) Zone d'arrêt :

L'avalanche s'arrête $10 \mathrm{~m}$ au-dessous de la console Al. Largeur du dépôt : $30 \mathrm{~m}$. L'avalanche a glissé en entraînant très peu la couche superficielle. Très peu de blocs, majorité de boules de $\phi 5$ à $10 \mathrm{~cm}$.

Epaisseur : 40 à $50 \mathrm{~cm}$ à l'arrêt.

\section{Résultats expérimentaux}

Les mesures de pression apportent les mêmes commentaires que pour les avalanches précédentes ainsi que le montre la figure 14.

Pression maximale : capteur haut 1,05 bar, capteur bas 0,60 bar.

Les vitesses, bien que perturbées par les blocs, donnent des vitesses maximales de l'ordre de $16 \mathrm{~m} / \mathrm{s}$. Les vitesses 
$P$
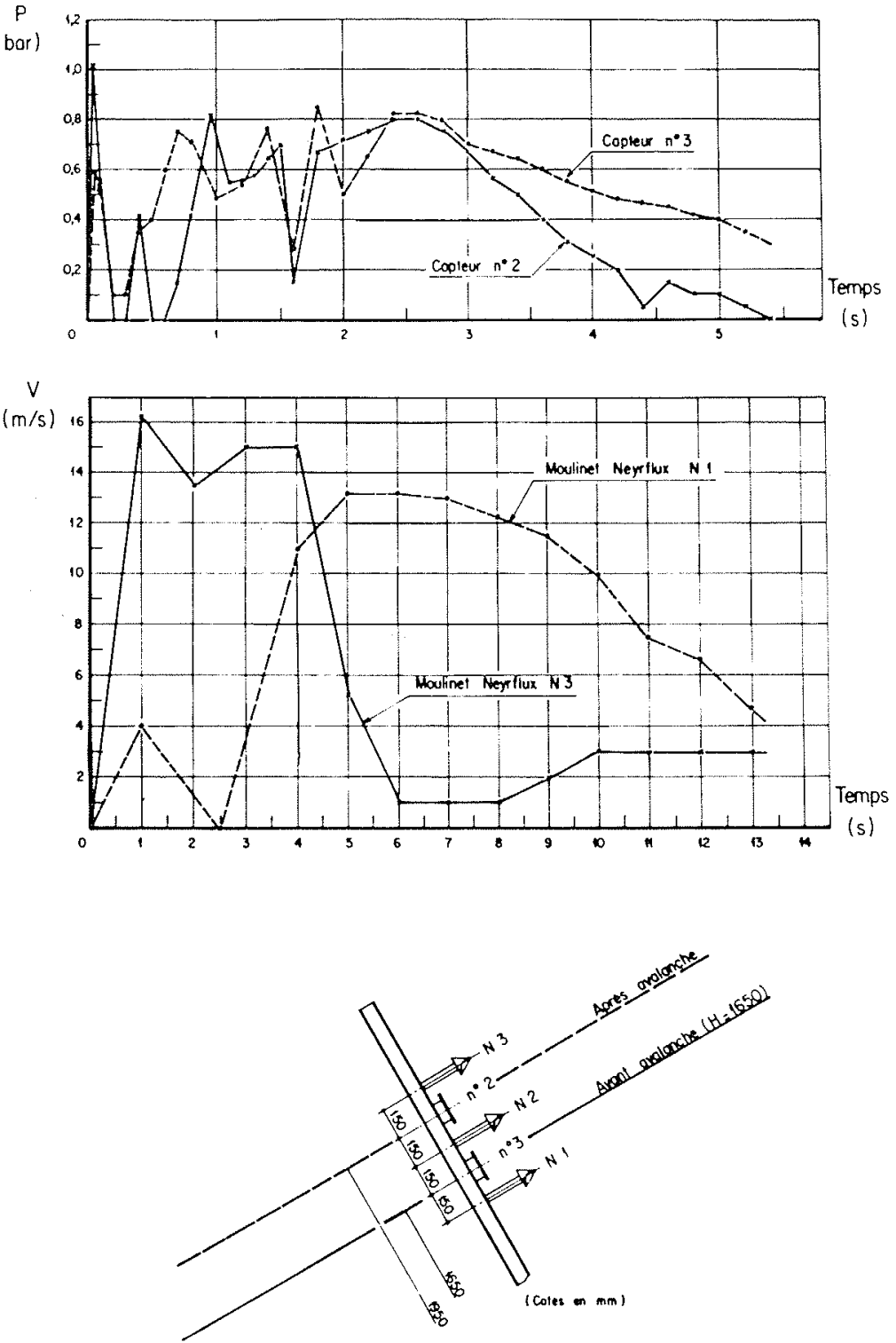

14/ Site du Lautaret le 22 Janvier 1975 - Couloir $n^{\circ} 2$ Console B

dépouillées sur le film du "Nikon $F_{2}$ " donnent des valeurs moyennes du front :

$$
18,7 \mathrm{~m} / \mathrm{s}<V_{m}<24,8 \mathrm{~m} / \mathrm{s}
$$

Coefficient d'augmentation des masses volumiques : 1,3 Augmentation de température : $+1,4^{\circ} \mathrm{C}$

\begin{tabular}{|c|c|c|c|c|}
\cline { 3 - 5 } \multicolumn{1}{c|}{} & \multicolumn{2}{c|}{$\begin{array}{c}\text { Masse volumique } \\
{\left[\mathrm{kg} / \mathrm{m}^{3}\right]}\end{array}$} & \multicolumn{2}{c|}{ Températures $\left[{ }^{\circ} \mathrm{C}\right]$} \\
\hline Niveaux & Avant & Après & Avant & Après \\
\hline Surface & & & $-4,6$ & $-2,0$ \\
$-10 \mathrm{~cm}$ & 160 & 288 & $-3,2$ & $-1,6$ \\
$-20 \mathrm{~cm}$ & - & - & $-4,0$ & $-2,0$ \\
$-30 \mathrm{~cm}$ & 196 & 304 & $-3,0$ & $-2,0$ \\
$-40 \mathrm{~cm}$ & - & - & $-2,0$ & $-2,1$ \\
$-50 \mathrm{~cm}$ & 320 & 312 & $-2,0$ & $-1,9$ \\
\hline$\rho$ moyen & 225 & 301 & $-3,3$ & $-1,9$ \\
\hline
\end{tabular}

Coeff. augm. $=1,3 \quad \Delta t^{\circ} \mathrm{C}=1,4^{\circ} \mathrm{C}$

\section{Avalanche du 12 Mars 1975}

\section{Conditions nivo-météorologiques}

\section{Etat du couloir :}

Epaisseur totale de neige au sommet du couloir : environ 250 à $280 \mathrm{~cm}$ dont $30 \mathrm{~cm}$ de neige récente.

L'épaisseur de neige au niveau de la console $B$ est d'environ $250 \mathrm{~cm}$ compte tenu des dépôts des avalanches antérieures.

Qualité de la neige de surface:

Neige sèche, légèrement ventée sur couche dure de vieille neige déjà métamorphosée.

Plan de glissement probable:

Partie supérieure de la couche compacte. L'épaisseur de neige mise en mouvement devrait être de l'ordre de 
$30 \mathrm{~cm}$ (neige de surface en plaque : masse volumique $\left.320 \mathrm{~kg} / \mathrm{m}^{3}\right)$.

Température surface neige : $-1,5^{\circ} \mathrm{C}$.

Température air : (non relevée).

2. caractéristiques de l'avalanche

a) Zone de départ :

Couche superficielle dont l'épaisseur est comprise entre 20 et $30 \mathrm{~cm}$.

b) Zone intermédiaire: Console $B$

Epaisseur de neige transportée : $39 \mathrm{~cm}$.

c) Zone d'arrêt: Manque de renseignements.

L'avalanche se produit avec écoulement au sol et nuage.

\section{Résultats Expérimentaux (fig. 15)}

Le capteur de pression haut n'a pas été atteint par l'avalanche, seul le capteur bas a "répondu", la faible épaisseur de neige en écoulement parsemée de blocs donne des pics assez importants sans surpression régulière.

Pression maximale du front : 1,2 bar

Les vitesses maximales sont de l'ordre de 16 à $18 \mathrm{~m} / \mathrm{s}$.

\begin{tabular}{|c|c|c|c|c|}
\cline { 3 - 5 } \multicolumn{1}{c|}{} & \multicolumn{2}{c|}{$\begin{array}{c}\text { Masse volumique } \\
{\left[\mathrm{kg} / \mathrm{m}^{3}\right]}\end{array}$} & \multicolumn{2}{c|}{ Températures $\left[{ }^{\circ} \mathrm{C}\right]$} \\
\hline Niveaux & Avant & Après & Avant & Après \\
\hline Surface & & & $-1,5$ & $-1,0$ \\
$-10 \mathrm{~cm}$ & 320 & 320 & $-3,0$ & $-1,0$ \\
$-20 \mathrm{~cm}$ & 160 & 320 & $-2,5$ & $-0,5$ \\
$-30 \mathrm{~cm}$ & 200 & 380 & $-1,5$ & 0 \\
$-40 \mathrm{~cm}$ & 340 & 320 & -1 & 0 \\
$-50 \mathrm{~cm}$ & - & - & $-0,5$ & 0 \\
$-60 \mathrm{~cm}$ & 340 & 360 & 0 & 0 \\
\hline$\rho$ moyen & 272 & 332 & $-1,4$ & $-0,4$ \\
\hline
\end{tabular}

Coeff. augm. $=1,2 \quad \Delta t^{\circ} \mathrm{C}=+1,0^{\circ} \mathrm{C}$
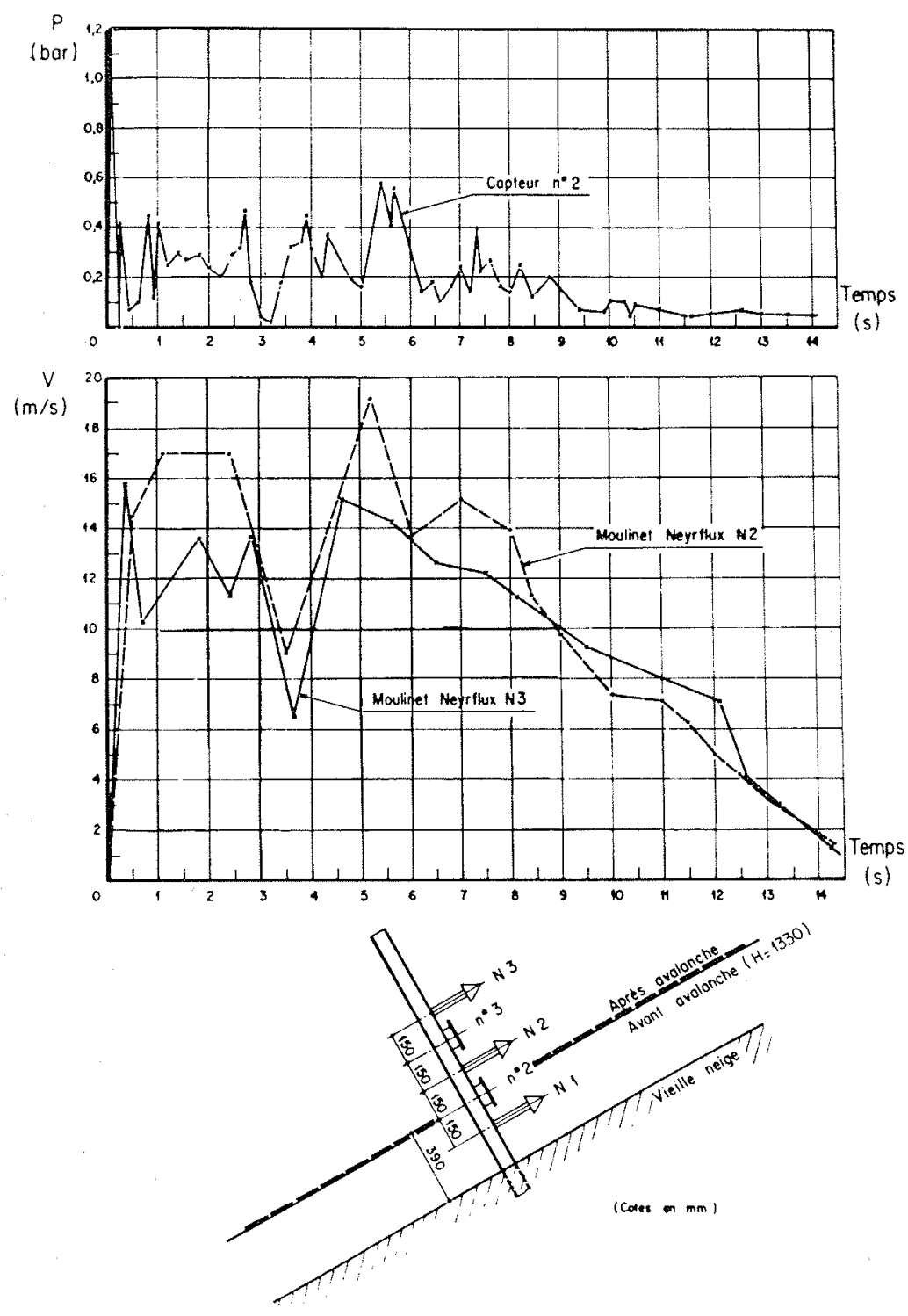

15/ Site du Lautaret le 12 Mars 1975 - Couloir $n^{\circ} 2$ Console B 


\section{Discussion}

Président : M.L. de CRECY

M. le Président remercie M. MuRA 'de son exposé improvisé au cours duquel, ne disposant pas du texte de l'équipe du C.E.N.G., il a dû se borner à commenter diverses diapositives et des tableaux de résultats.

Un intéressant film sur les expériences de déclenchement artificiel des avalanches au Lautaret est ensuite présenté. A ce propos. M. le Président apporte les précisions ci-après:

Dans le film, le déclenchement de l'explosion est assuré par une "mèche lente". Ce procédé -... tout provisoire .... a été remplacé par un déclenchement électrique utilisant comme conducteur le câble lui-même (en prenant toutes précautions contre l'é. ventuels courants induits ... par la foudre par exemple)".

\section{Abstract \\ "Avalanche dynamics" data from artificial avalanche experiments at the Lautaret Pass in the French Alps}

Results of tests carried out at an experimental site on the Lautaret Pass during the 1974/75 snow season are described. Two avalanche corridors are fitted with instrumentation ; one starts at an altitude of $2,320 \mathrm{~m}$ and ends $90 \mathrm{~m}$ below, and the other drops $210 \mathrm{~m}$ from an altitude of $2,340 \mathrm{~m}$. Snow depths vary from $0.7 \mathrm{~m}$ to $2.8 \mathrm{~m}$.

The avalanches are set off by explosives positioned by a conveyor cable and fired by a special safety unit designed by "Laboratoire A.S.P".

Avalanches were triggered in powder snow on the $22 \mathrm{nd}$ and 31 st January 1975 and in powder and sheet snow on the 12 th March 1975.

Test measurements are as follows :

1) Particle velocities in the avalanche, using hydraulic currentmeters and cinematographic equipment. Average velocity is around $7 \mathrm{~m} / \mathrm{s}$ and peak velocities up to $16-18 \mathrm{~m} / \mathrm{s}$.

2) Impact pressure, using strain-gauges. Peak values are recorded behind the avalanche front, ranging from 0.6 bar to $1.3 \mathrm{bar}$.

3) Specific mass of the flowing snow, by gamma attenuation and comparison with gravimetric measurement data obtained before and after the avalanche. The coefficient of increase is approximately
1.3. Measured data for various depths are shown on tables.

4) Specific mass of the aerosol, by gamma attenuation (cesium 137 , activity $37 \mathrm{mCi}$, horizontal distance $2.24 \mathrm{~m}$ ) at $5 \mathrm{~m}$ above the bottom. However, the proportion of aerosols in the avalanche is too small for the measurements to carry significant weight.

5) Snow temperature in the avalanche, using platinum tempe rature probes. Temperature rises of $1.6^{\circ} \mathrm{C}, 1.4^{\circ} \mathrm{C}$ and $1.0^{\circ} \mathrm{C}$ have been recorded. Snow temperatures at various depths are listed on tables.

6) Aerosol velocity, using a standard French Meteorological Service Type W106 propeller anemometer.

7) Avalanche front velocity, depth and size of the avalanche. These are determined by photography at 4 frames/second with reference to appropriate markers along the corridor. With this equipment, an avalanche can be observed over much of its length.

8) Electrostatic charges carried by the avalanche. Attempts have been made to measure these with a Rotschild electrometer a few metres from the edge of the avalanche, but so far without significant results.

Data obtained for twelve avalanches in 1973, 1974 and 1975 are listed in a general table. 